\title{
Assessment of emergency trolley drugs in a children's hospital. Before and after study on an educational intervention
}

\author{
María I. Fernández Achával, M.D. ${ }^{a}$, Luis F.Mammi, M.D. ${ }^{a}$, Nicolás Fortini Cabarcos, M.D. ${ }^{a}$, \\ Gonzalo G. Guiñazú, M.D. ${ }^{a}$, Cecilia A. Mercedes Robledo, M.D. ${ }^{a}$, Julia Dvorkin, M.D., \\ Ezequiel Monteverde, M.D. ${ }^{b}$, Carolina Pascual, M.D. ${ }^{c}$, Christian Elías Costa, M.D. ${ }^{d}$ and \\ Eduardo O. Vainstein, M.D.
}

\begin{abstract}
Introduction. The approach to pediatric emergencies requires specialized resources, and medication errors are common.

Objectives. To describethe contents of emergency trolleys(ETs)in a children's hospital and compare them after an educational intervention.

Methods. The ETs from 9 hospitalization wards were included. A checklist of 30 drugs was developed. ETs were assessed by determining whether drugs were present or absent and their amount. An educational intervention was conducted and assessments were repeated 30 and 90 days after the intervention.

Results. The baseline measurement indicated an overall ratio of adequate drugs of $43.9 \%$ (95\% confidence interval [CI]: 38.4-49.4) with a variation among ETs from $29 \%$ to $54.8 \%$, and from $15 \%$ to $85 \%$ among drug groups. At 30 days, the adequate ratio reached $70.3 \%$ (95\% CI: 65.275.4), with a wide variation among the different ETs and drug groups (from $51.6 \%$ to $93.5 \%$ and from $50 \%$ to $95 \%$, respectively). At 90 days, the percentages were similar. The comparison between the first and second measurement showed an improvement in all ETs (range: $3.2 \%-45.1 \%$ ), odds ratio: 3.73 (95\% CI: 2.5-5.6), $p<0.001$. Results were similar between the second and third measurement.

Conclusions. Thebaseline measurement showed a low level of adequate ET drugs. After the intervention, this improved significantly and was maintained during the studied period.

Key words: instrumentation, medical emergencies, pediatrics, cardiopulmonary resuscitation.
\end{abstract}

a. Clinical Pediatrics, Teaching and Research Committee, Hospital de Niños Dr. Ricardo Gutiérrez, Autonomous City of Buenos Aires (CABA).

b. Intensive Care Unit, Hospital de Niños Dr. Ricardo Gutiérrez, CABA.

c. Unit 5, Hospital de Niños Dr. Ricardo Gutiérrez, CABA.

d. Nephrology, Teaching and Research Committee, Hospital de Niños Dr. Ricardo Gutiérrez, CABA.

Argentina

E-mail address:

María I. Fernández

Achával, M.D.:

inesfachaval@gmail.com

http: / / dx.doi.org/10.5546/ aap.2020.eng.234

To cite: Fernández Achával MI, Mammi LF, Fortini Cabarcos N, Guiñazú GG, et al. Assessment of emergency trolley drugs in a children's hospital. Before and after study on an educational intervention. Arch Argent Pediatr 2020;118(4):234-239.

\section{INTRODUCTION}

Emergencies that require resuscitation with drugs are uncommon in pediatrics. One of such emergencies is cardiorespiratory arrest (CRA), which has an important morbidity and mortality. In the USA, it accounts for an incidence of 8-20/100 000 children per year and $2-5.5 \%$ of admissions to pediatric intensive care units. It has been estimated that less than $8 \%$ of children seen due to a CRA outside of a hospital setting will survive the event, whereas up to $27 \%$ will survive if it occurs within a hospital. In recent years, survival among these patients has improved, partly due to regular updates and the wide dissemination of advanced cardiopulmonary resuscitation (CPR) guidelines.

The approach to pediatric emergencies requires specialized resources, including equipment, drugs, and trained staff. ${ }^{1}$ Materialand medication-related errors during CPR are common and may have an impact on patients' morbidity and mortality..$^{5,6}$ For this reason, its use should be based on an organized, effective system. ${ }^{6}$ Health care facilities should have the necessary equipment available and adequately trained health care providers. In this context, an indispensable health care component is the emergency trolley (ET). This type of cart helps to organize the material used during an emergency and is placed in the hospital areas where an emergency is most likely to occur. It should be reserved for use in the situations established in emergency protocols 
and use, replacement, and maintenance procedures should be implemented. ${ }^{7-9}$

In 1995, the American Academy of Pediatrics published guidelines that established the facility characteristics necessary for pediatric emergency care. These guidelines defined the importance of resource organization to provide an optimal and timely response and achieve adequate health care levels for critically-ill patients. ${ }^{1}$ In 2003, a new recommendation was published indicating that drugs routinely used in emergency situations should be accessible and clearly labeled based on some sort of visual reference to estimate the drug dose according to weight and/or height. All health care providers should know where every element needed for emergency care is located; and ongoing education is very important for a correct ET use. ${ }^{9,10}$

Although international guidelines provide recommendations on the desirable ET equipment, in our setting, ET contents are not homogeneous. Sometimes, materials correspond to other requirements, in addition to typical CPR or other emergency needs, resulting from the activities performed where the ET is located. ${ }^{11}$ Occasionally, it is not clearly defined who is responsible for the replacement and maintenance of its contents. At the hospital where we carried out this study there is no checklist for an adequate drug provision during an emergency. For these reasons, it is important to conduct a study to describe the current situation of ETs and, thus, evidence the problem in our setting and propose possible solutions.

\section{OBJECTIVES}

The primary objective of this study was to do an initial descriptive analysis of ET contents in different areas of a children's hospital by comparing them against a previously designed checklist. The secondary objective was to assess the effectiveness of an educational intervention provided to the staff responsible for ET supply and maintenance and compare its contents after 30 and 90 days.

\section{POPULATION AND METHODS}

An analytical, prospective, quasi-experimental, non-controlled (before and after) study was conducted in the clinical hospitalization wards dependent on the Department of Medicine of a tertiary care children's hospital in the Autonomous City of Buenos Aires between August 2018 and March 2019.
Based on different publications, drugs considered indispensable in an ET were determined and their minimum amount was defined. ${ }^{1-3}$ To this end, the possibility of performing advanced CPR for 30 minutes according to current protocols was considered, together with the necessary drug amounts for other emergencies, which was agreed upon with the hospital's departments of pharmacy and nursing and based on drug availability. A checklist was developed based on this information (Table 1). All wards where there was an ET were included. The neurosurgery ward was excluded because it was being remodeled. Units where emergencies were managed in a standardized manner, such as the intensive care unit, the intermediate care unit, and the outpatient emergency room, were also excluded.

First of all, 4 trained investigators examined the ETs from 9 hospitalization wards, with the authorization of the Department of Medicine, but not informing the heads of Nursing and Clinical Medicine of each ward: 4 Clinical Pediatrics wards and 5 specialty wards (Hepatology, Pulmonology, Nephrology, and Infectious Diseases [2]), using the previously designed checklist. Each ward had a single ET, whose preparation, maintenance, and verification were the responsibility of nurse managers. For each ET, the presence or absence of drugs was determined. If present, drug's expiry date or sufficient/insufficient amount were also checked.

Once the initial data were collected, the staff responsible for the ET at each of the assessed wards was trained. Training consisted in 2 face-to-face sessions that lasted 1 hour each, during which the importance of having the corresponding material to perform basic and advanced high-quality CPR and other emergencies was described. The checklist was delivered and staff were trained on how to use it. At 30 and 90 days after the intervention, all ETs were reviewed again at a similar time as the first assessment. Visits were not reported in advance to the medical staff or the nurses of each ward so as to prevent outcome bias.

Before data collection, a standard operating procedure manual was developed. The manual detailed checklist coding, adequate data input, and how to note absent or inaccurate information. Drugs were divided into 9 categories for analysis (Table 2). Two trained, blinded operators were in charge of double data entry. Input data were compared and no differences were observed. 
A descriptive analysis was performed in the first place. For each hospitalization ward and for each of the three measurements, the percentage of adequate items was determined, together with the $95 \%$ confidence interval (CI), and the reasons for items being considered inadequate. Measurements were then compared and the McNemar test for paired data was done to estimate effect sizes and the level of significance of observed differences. The statistical software package $R$ was used.

Before the conduct of this study, authorization was requested to the heads of the participating units, the Head of the Department of Medicine, and the hospital's Ethics Committee and Research and Teaching Committee. In addition, the confidentiality of all data collected in the data collection sheets was warranted.

\begin{tabular}{|c|c|c|c|}
\hline \multicolumn{4}{|c|}{ EMERGENCY TROLLEY DRUG CHECKLIST } \\
\hline \multicolumn{4}{|l|}{ UNIT/DEPARTMENT: } \\
\hline \multicolumn{4}{|l|}{ DATE OF REVIEW: } \\
\hline \multicolumn{4}{|l|}{ SEAL No./CODE: } \\
\hline \multicolumn{4}{|c|}{$\begin{array}{c}\text { The expiry date of each vial should be checked. Those that have expired and/or will expire in the next month } \\
\text { should be discarded. }\end{array}$} \\
\hline Drugs & $\begin{array}{l}\text { Minimum required } \\
\text { amount }\end{array}$ & Expiry date & Checked \\
\hline Adenosine & 2 vials & & \\
\hline Amiodarone & 2 vials & & \\
\hline \begin{tabular}{|l|} 
Adrenaline \\
\end{tabular} & 10 vials & & \\
\hline Atropine & 3 vials & & \\
\hline Sodium bicarbonate & $100 \mathrm{mEq} \times 2$ & & \\
\hline \begin{tabular}{|l} 
Neuromuscular blocking agents (vecuronium) \\
\end{tabular} & $10 \mathrm{mg}$ & & \\
\hline Potassium chloride $3 \mathrm{M}$ & 4 vials & & \\
\hline Dopamine & 5 vials & & \\
\hline Phenytoin = diphenylhydantoin & 15 vials & & \\
\hline Calcium gluconate $10 \%$ & 2 vials & & \\
\hline Lidocaine & 3 vials & & \\
\hline Naloxone & 5 vials & & \\
\hline Magnesium sulphate $25 \%$ & 2 vials & & \\
\hline Fentanyl & 2 vials & & \\
\hline Diazepam & 2 vials & & \\
\hline Diphenhydramine & 2 vials & & \\
\hline Intravenous antipyretic agents: dipyrone/ibuprofen & 2 vials & & \\
\hline \begin{tabular}{|l|} 
Flumazenil \\
\end{tabular} & 2 vials & & \\
\hline \begin{tabular}{|l|} 
Furosemide \\
\end{tabular} & 5 vials & & \\
\hline Haloperidol & 2 vials & & \\
\hline Hydrocortisone or dexamethasone or methylprednisolone & 2 vials & & \\
\hline Insulin & 1vial REFRIGERATED & & \\
\hline Ketamine & 1 vial & & \\
\hline Lorazepam & 4vials REFRIGERATED & & \\
\hline Midazolam & 3 vials & & \\
\hline \begin{tabular}{|l|} 
Dextrose solution \\
\end{tabular} & 2 sachets & & \\
\hline Saline solution $500 \mathrm{~mL}$ & 2 sachets & & \\
\hline Saline solution & 10 vials & & \\
\hline Distilled water & 10 vials & & \\
\hline
\end{tabular}

To develop the checklist, indispensable ET drugs were determined and the minimum required amount was defined. It was based on the bibliography and agreed upon with the hospital's departments of pharmacy and nursing based on drug availability and accessibility. mEq: mill equivalent; mL: milliliter; M: molar. 


\section{RESULTS}

The first measurement showed a low overall ratio of adequate drugs (i.e., not expired or

TABLE 2. Emergency trolley drug grouping based on their use in an emergency

\begin{tabular}{|c|c|}
\hline Group & Drug \\
\hline Antiarrhythmics & $\begin{array}{l}\text { Amiodarone } \\
\text { Atropine } \\
\text { Lidocaine }\end{array}$ \\
\hline Anticonvulsant agents & $\begin{array}{l}\text { Diazepam } \\
\text { Diphenylhydantoin } \\
\text { Phenobarbital } \\
\text { Lorazepam }\end{array}$ \\
\hline Antidotes & $\begin{array}{l}\text { Flumazenil } \\
\text { Naloxone }\end{array}$ \\
\hline Diuretics & $\begin{array}{l}\text { Furosemide } \\
\text { Mannitol }\end{array}$ \\
\hline Anaphylaxis drugs & $\begin{array}{l}\text { Diphenhydramine } \\
\text { Hydrocortisone } \\
\text { dexamethasone }\end{array}$ \\
\hline OTI drugs & $\begin{array}{l}\text { Fentanyl } \\
\text { Ketamine } \\
\text { Midazolam } \\
\text { Vecuronium }\end{array}$ \\
\hline Electrolyte solution & $\begin{array}{l}\text { Bicarbonate } \\
\text { Potassium chloride } \\
\text { Calcium gluconate } \\
\text { Magnesium soleplate } \\
\text { Saline solution vials } \\
\text { Distilled water vials } \\
\text { Ringer's lactate solution } \\
\text { Saline solution x } 500 \mathrm{~mL}\end{array}$ \\
\hline Vasopressors & $\begin{array}{l}\text { Adrenaline } \\
\text { Dopamine }\end{array}$ \\
\hline Other & $\begin{array}{l}\text { Dipyrone } \\
\text { Insulin } \\
\text { Dextrose solution }\end{array}$ \\
\hline
\end{tabular}

OTI: oro-tracheal intubation. insufficient or absent) of $43.9 \%$ (95\% CI: 38.4-49.4), with a variation among ETs from $29 \%$ to $54.8 \%$, and from $15 \%$ (diuretics) to $85 \%$ (antidotes) when drug groups were considered (Figure 1). The second measurement after 30 days showed an adequate ratio of $70.3 \%$ (95 \% CI: 65.2-75.4), with an even greater variation among ETs (from $51.6 \%$ to $93.5 \%$ ) and from $50 \%$ (vasopressors) to $95 \%$ (antidotes) among drug groups. The third measurement after 90 days showed similar results to the second one: an adequate ratio of $69.4 \%$ (95\% CI: 64.3-74.5), also with a similar variation among ETs (from $51.6 \%$ to $93.5 \%$ ) and, among drug groups, from $45 \%$ (antiarrhythmics) to $90 \%$ (antidotes). The reasons why items were considered inadequate at the three measurements are shown in Table 3.

The comparison between the first and second measurement showed an improvement in all ETs (range: $3.2 \%-45.1 \%$ ), with a statistically significant difference between both: odds ratio (OR): 3.73 (95\% CI: 2.5-5.6), $p<0.001$. We also assessed whether ETs with a greater initial ratio of adequate items had a higher improvement proportion based on the correlation between the ratio of adequate items and the size of change, and Spearman's coefficient $r$ was observed to be -0.29 .

The comparison between the second and the third measurement showed similar results: OR: 1.1 (95\% CI: 0.7-1.8), $p=0.71$, with a reduction in adequate items in 3 ETs (range: $3.2 \%-9.7 \%$ ), no changes in 1 , and improvement in the other 5 (range: $3.2 \%-22.6 \%$ ). The assessment between the first and third measurement showed a significant and sustained improvement during the study period: 1 ET remained stable and the

Figure 1. Assessment of adequate items in the first measurement. Percentage of adequate drugs by category

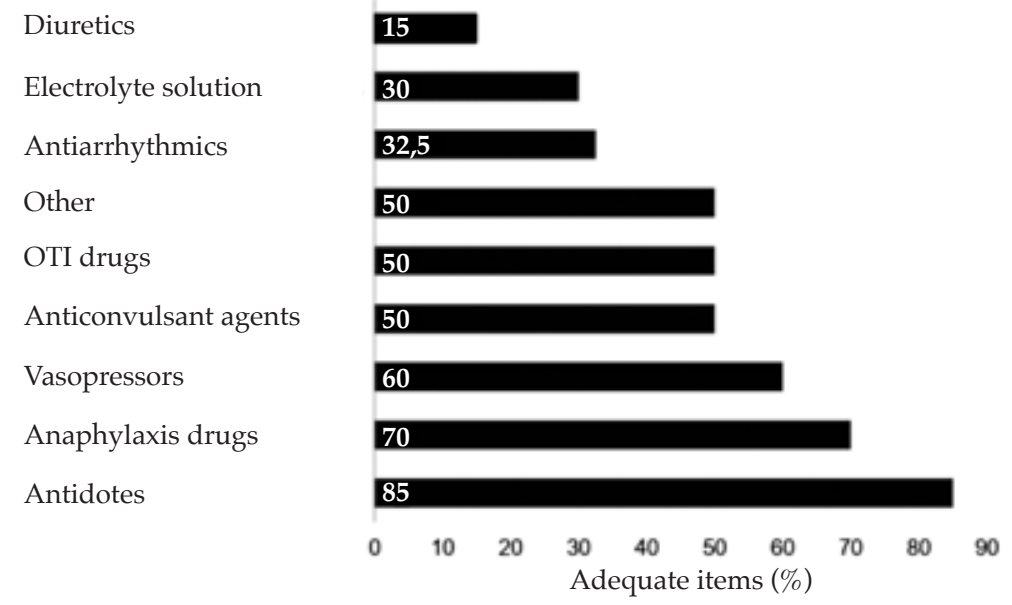

OTI: oro-tracheal intubation. 
rest improved (range: $19.4 \%-61.3 \%$ ), OR: 3.55 (95\% CI: 2.4-5.3), $p<0.001$ (Figure 2).

\section{DISCUSSION}

According to the USA Agency for Healthcare Research and Quality, improvements in health care quality are reached through systematic thinking, data analysis, and team work to achieve better results and processes and reduce errors that may affect patients and their families. ${ }^{12,13}$ One of the domains of quality is safety, defined as the reduction of risk of unnecessary harm to an acceptable minimum. ${ }^{14}$

Several studies tried to analyze the most common errors occurred during emergencies. ${ }^{6,16-19}$ It is evident that, in a stressful situation, as a CRA,

TABle 3. Classification of inadequate items. Differences among the three measurements for inadequate items classified as absent, insufficient, and expired

\begin{tabular}{lclc}
\hline & $\begin{array}{c}\text { Baseline measurement } \\
\text { n (\%) }\end{array}$ & $\begin{array}{l}\text { 30 days } \\
\text { n (\%) }\end{array}$ & $\begin{array}{c}\mathbf{9 0 ~ d a y s} \\
\mathbf{n}(\%)\end{array}$ \\
\hline Absent & $107(62.6)$ & $38(46.3)$ & $24(43.6)$ \\
Insufficient & $37(21.6)$ & $25(30.5)$ & $27(49.1)$ \\
Expired & $27(15.8)$ & $19(23.2)$ & $4(7.3)$ \\
Total & $171(100)$ & $82(100)$ & $55(100)$ \\
\hline
\end{tabular}

n: number of measurements. drug administration errors may occur, and an inadequate supply may be the first link in the chain of errors. For this reason, a standardized approach generates a safer environment, especially if the user is not familiarized with the management. ${ }^{19}$ This study evidenced a considerable overall improvement in ET drugs after an educational intervention. All ETs in the studied wards showed a significant improvement, although results were not homogeneous among the different hospitalization wards. It is worth noting that the assessment results were sustained in the study period, and no significant differences were observed between the second and third measurement. In addition, the wards with the higher ratios of adequate drugs were the ones that changed their initial contents the least.

Fierro Rosón et al., assessed ET contents and functioning in a Spanish hospital and described deficiencies that may have compromised or hindered CPR effectiveness, and only a minority of ETs were adequately equipped. ${ }^{12}$ Errors in the management of emergencies, such as CPR, caused by resource use problems may lead to a sentinel event (defined as that causing a severe adverse event). ${ }^{13-15}$ This type of event evidences a system weakness that requires immediate analysis and resolution. Because of this, it is important to

FIGURE 2. Comparison among the three measurements. Adequate item ratio over the total for the three measurements. Odds ratio for the difference between the first and second measurement and between the second and third measurement

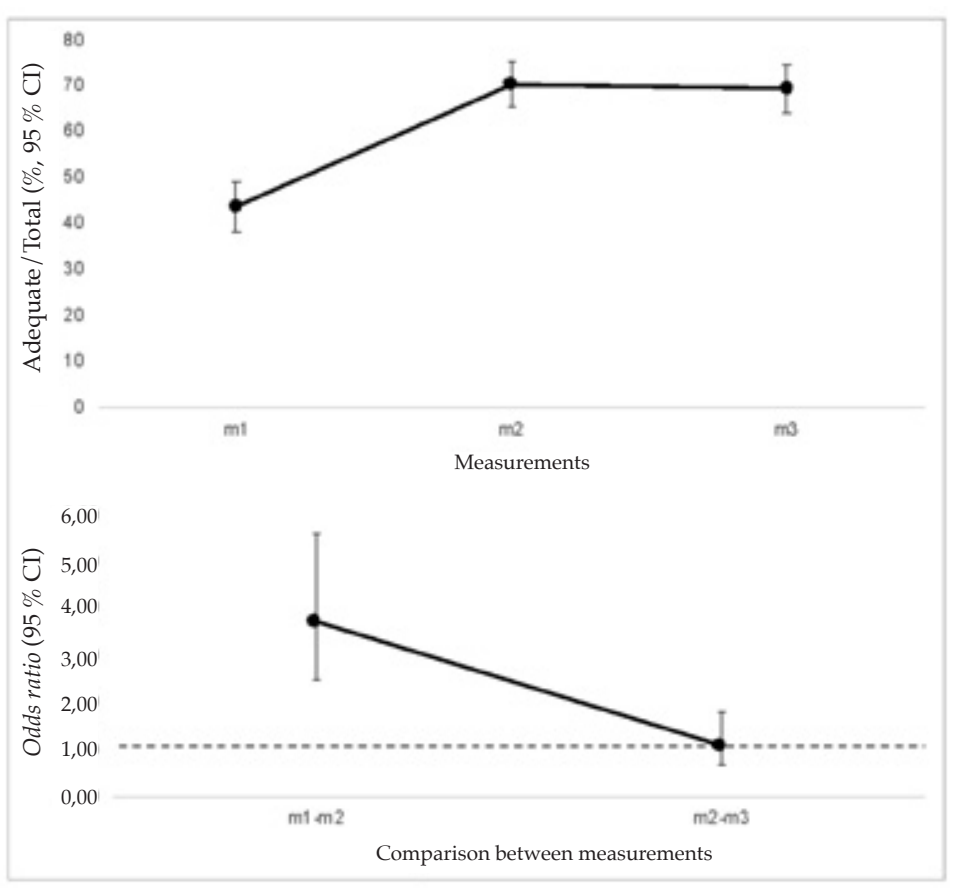

CI: confidence interval; m: measurement. 
implement interventions targeted at this problem in our setting.

In addition, it is worth noting that it is critical for health care services to have their staff trained regularly, even in units where the frequency of $\mathrm{CPR}$ or other emergencies is low. According to international recommendations, one of the steps in survival improvement consists in the possibility of having continuously trained staff available because skills deteriorate months after being acquired. ${ }^{2}$

This study had certain weaknesses. ET contents were analyzed exclusively in relation to drugs. Multiple other materials, such as the devices necessary for airway management or defibrillators, are also a critical part of ETs. These items were not analyzed because, given the format of our ETs (small cases or trolleys with few drawers and open racks), many of them are found in procedure rooms or next to the ETs, not precisely in the ETs. We intend to include them in future measurements. In addition, given ET heterogeneity, it was not possible to assess drug distribution inside the corresponding compartments.

It is known that the protocols for ET equipment vary among the different public and private facilities in the Autonomous City of Buenos Aires, and that there are no regulations in relation to them. This may weaken the external validity of our study. In addition, the areas where CPR is more common, like the emergency department, the intensive care unit or the intermediate care unit, were excluded from this analysis because of structural differences with the other wards.

One of the strengths of this study is that it detected a major deficiency in health care activities, such as an inadequate ET equipment. In addition, it has been demonstrated that establishing an ET protocol together with an educational intervention may help to achieve significant and sustained improvements. We propose to continue making measurements over time and to extend interventions to other hospital areas to try and standardize ET contents.

\section{CONCLUSIONS}

The baseline measurement showed a low level of adequate ET drugs. Such level was heterogeneous among the different hospitalization wards. After the intervention, the ratio of adequate drugs improved significantly and was maintained during the studied period.

\section{REFERENCES}

1. Committee on Pediatric Emergency Medicine. American Academy of Pediatrics. Guidelines for Pediatric Emergency Care Facilities. Pediatrics. 1995; 96(3 Pt 1):526-37.
2. MonsieursKG,NolanJP, BossaertLL, GreifR, etal.European Resuscitation Council Guidelines for Resuscitation 2015 Section 1. Executive summary. Resuscitation. 2015; 95:1-80.

3. NeumarRW, ShusterCM, Callaway CW, GentLM, etal.Part 1: Executive Summary: 2015 American Heart Association Guidelines Update for Cardiopulmonary Resuscitation and Emergency Cardiovascular Care. Circulation. 2015; 132(18 Suppl 2):s315-67.

4. Nadkarni VM, Larkin GL, Peberdy MA, Carey SM, et al. First documented rhythm and clinical outcome from inhospital cardiac arrest among children and adults. JAMA. 2006; 295(1):50-7.

5. Meaney PA, Nadkarni VM, Cook EF, Testa M, et al. Higher survival rates among younger patients after pediatric intensive care unit cardiac arrests. Pediatrics. 2006; 118(6):2424-33.

6. Flannery AH,ParliSE.MedicationErrorsinCardiopulmonary Arrest and Code-Related Situations. Am J Crit Care. 2016; 25(1):12-20.

7. Regalado Becerra CA, Segura Vega J, Órnelas Aguirre JM. Evaluación de conocimientos y equipamiento en los carros rojos para la reanimación cardiopulmonar en una unidad de tercer nivel de atención. Medicrit. 2008; 5(2):63-73.

8. López-Herce Cid J, Rodríguez Núñez A, Carrillo Álvarez A, ZeballosSarrato G, et al. Recomendaciones de expertos sobre material del carro y mochila de reanimación cardiopulmonar pediátrica y neonatal. An Pediatr (Barc). 2018; 88(3):173. e1-173.e7.

9. Bowden T, SmithD. An overview of adult cardiopulmonary resuscitation equipment. Nurs Stand. 2017; 31(23):54-63

10. Castillo J, García-Guasch R. Organización y enseñanza de la reanimación cardiopulmonar. Rev Esp Anestesiol Reanim. 1999; 46:183-5.

11. Sigrest TD; American Academy of Pediatrics Committee on Hospital Care. Facilities and equipment for the care of pediatric patients in a community hospital.Pediatrics.2003; 111(5 Pt 1):1120-2.

12. Fierro Rosón J, Ruiz Bailén M, Peinado Rodríguez J, Ramos cuadra JA, et al. Evaluación del contenido y funcionamiento delos carros de reanimación cardiopulmonar deun hospital. Med Intensiva. 2003; 27(6):399-403.

13. Donabedian A. The Definition of Quality and Approaches to its Assessment. Explorations in Quality Assessment and Monitoring, Vol. 1. Chicago, IL: Health Administration Press; 1980.

14. Organización Mundial de la Salud, Patient Safety. Más que palabras. Marco Conceptual dela Clasificación Internacional para la Seguridad del Paciente Versión 1.1. Informe técnico 2009. [Accessed on: April 26 $6^{\text {th }}, 2019$ ]. Available at: www. who.int/patientsafety/implementation/taxonomy/icps_ technical_report_es.pdf.

15. Joint Commission International. Standard QPS.7: The hospital uses a defined process for identifying and managing sentinel events. In: Joint Commission International Accreditation standards for hospitals. $5^{\text {th }}$ ed. Oakbrook Terrace, IL: Joint Commission Resources: 2013;145.

16. Subcomisión de Calidad en Salud y Seguridad del Paciente Sociedad Argentina de Pediatría, Sabio Paz V, Panattieri N, Godio C, et al. Seguridad del paciente: glosario. Arch Argent Pediatr. 2015; 113(5):469-72.

17. Agarwal S, Swanson S, Murphy A, Yaeger K, et al. Comparing the utility of a standard pediatric resuscitation card with a pediatric resuscitation cart based on the Broselow tape: a randomized, controlled, crossover trial involving simulated resuscitation scenarios. Pediatrics.2005; 116(3):e326-33.

18. Bergon-Sendin E, Pérez-Grande MdC, Lora-Pablos D, Melgar-Bonis A, et al. Auditorías de seguridad en tiempo real en una unidad neonatal. An Pediatr (Barc). 2017; 87(3):148-54.

19. Griffiths SE, Boleat E, Goodwin A, Sheikh A, et al. Efficacy of paediatric anaesthetic trolleys: A call for a basic standard and layout. Int J Risk Saf Med. 2015; 27(4):201-7. 